\title{
Mission de David Van Reybrouck
}

\section{Maëline Le Lay}

Édition électronique
URL : https://journals.openedition.org/coma/563

DOI : $10.4000 /$ coma.563

ISSN : 2275-1742

\section{Éditeur}

Institut des textes \& manuscrits modernes (ITEM)

\section{Référence électronique}

Maëline Le Lay, « Mission de David Van Reybrouck », Continents manuscrits [En ligne], 4 | 2015, mis en ligne le 15 mars 2015, consulté le 29 août 2022. URL : http://journals.openedition.org/coma/563 DOl : https://doi.org/10.4000/coma.563

Ce document a été généré automatiquement le 29 août 2022.

\section{(c) (i) ()}

Creative Commons - Attribution - Pas d'Utilisation Commerciale - Pas de Modification 4.0 International - CC BY-NC-ND 4.0

https://creativecommons.org/licenses/by-nc-nd/4.0/ 


\title{
Mission de David Van Reybrouck ${ }^{1}$
}

\author{
Maëline Le Lay
}

\section{NOTE DE L'AUTEUR}

Mise en scène de Raven Rüell avec Bruno Vanden Broecke, une création du KVS (Koningklijke Vlaamse Schouwburg) de Bruxelles. Spectacle donné au TNBA, Théâtre National de Bordeaux en Aquitaine, du 21 au 25 janvier 2014.

Ce texte est antérieurement paru en version abrégée dans Agôn. Revue des arts de la scène ; URL : http://agon.ens-lyon.fr/index.php?id=3024.

1 Créée en 2007 au KVS (Koningklijke Vlaamse Schouwburg, le Théâtre Royal Flamand) et rejouée six ans plus tard en 2013, la pièce Mission s'inscrit aujourd'hui dans le sillage de l'œuvre majeure de David Van Reybrouck, best-seller de la rentrée 2012, Congo. Une histoire. Il paraît évident, en effet, que le succès de cette pièce ${ }^{2}$ qui lui vaut aujourd'hui une tournée européenne, est à lire à l'aune du succès éditorial et médiatique de Congo ${ }^{3}$, tant le sujet de Mission présente a priori peu d'attraits pour le spectateur lambda : le monologue d'un père blanc, Grégoire, qui raconte sa vie et sa vocation, de sa jeunesse à ses quarante-huit années passées en République démocratique du Congo, de 1959 à 2007.

2 Y sont évoqués dans un ordre chronologique et avec un effet de coq-à-l'âne suggérant efficacement la conversation, l'éducation reçue de ses parents et à l'école, ses loisirs et son amour de jeunesse, sa formation ecclésiastique suivie de son départ pour le Congo. Il nous entretient de sa vie de missionnaire passée dans l'Est du pays, du Nord Katanga au Kivu, de son travail, des bonnes œuvres accomplies au cours de ses différentes affectations, de ses amitiés, des guerres qu'il a vécues, de la terreur et des exactions qu'il a vu commettre dans cette région sinistrée par les conflits. Cette anomie meurtrière, David Van Reybrouck la rend plus palpable encore en intégrant, dans la trame du monologue, le témoignage d'une femme otage d'un groupe rebelle et réduite à l'esclavage sexuel, témoignage qui a pour effet de situer le spectacle dans une criante et tragique actualité. En dépit de ces ruptures et de son cheminement non-linéaire, le 
récit demeure fluide et bien rythmé par de fréquents effets d'oralisation de la langue qui donnent à entendre un français congolais.

3 La cascade de toponymes qui émaillent le discours monologique d'un bout à l'autre semble pourtant menacer la capacité du spectacle à retenir durablement l'attention d'un spectateur qui ignorerait tout de ce pays, ou du moins à faire en sorte qu'il puisse se sentir concerné. En regard de cette toponymie par endroits envahissante, cet important cadrage géographique et géopolitique tranche avec la quasi absence des langues que mentionne régulièrement le Père Grégoire - lingala, kitembo et swahili - et dont le texte original est pourtant truffé au point d'intégrer une conversation téléphonique en swahili immédiatement suivie de sa traduction en français dans le texte original.

4 Mais, l'essentiel n'est pas là. Qu'il soit ou non familier de l'histoire et de la géographie de la République démocratique du Congo, il me semble que le spectateur ne peut qu'être convaincu par le personnage qui, quoique seul en scène d'un bout à l'autre de la pièce, fait montre d'un charisme extraordinaire. L'interprétation remarquable de Bruno Vanden Broecke donne véritablement du souffle et de l'ampleur au texte tout en respectant le pari - risqué - de la mise en scène imaginée par l'auteur qui est minimaliste au possible (le texte original est dépourvu de toute didascalie gestuelle). Le Père Grégoire reste donc statiquement posté derrière un pupitre pendant les deux heures que dure le spectacle.

5 Cette tribune d'orateur est le premier des partis-pris scénographiques de Raven Ruëll. Là où David Van Reybrouck amorçait sa pièce par le récit du personnage sans introduction aucune, le metteur en scène a ajouté une sorte de préambule, court moment au cours duquel le personnage se présente en expliquant qu'au cours de son séjour de vacances en Europe, on lui a demandé de venir parler de son expérience de missionnaire. C'est dans ce cadre que nous, spectateurs, le rencontrons. Son récit sera ainsi ponctué par les allers et retours entre le Congo et la Belgique, sa vie là-bas et les activités menées ici en Europe au cours de son séjour.

6 La mise en scène obéit donc à une grande sobriété pendant toute la pièce jusqu'à l'orage final annoncé par un formidable coup de tonnerre qui prend le spectateur par surprise et qui semble déclenché par le cri de désespoir et de colère du personnage dont l'humeur était jusque-là égale : sincère et plutôt débonnaire, souvent assez gaie, même dans le sarcasme. "Dieu! » tonne-t-il subitement, en proie à une violente crise de doute qui ébranle sa foi. À peine le nom sacré proféré qu'une pluie diluvienne se met à tomber, le trempant des pieds à la tête. Cet effet spécial qui aurait pu donner du corps à son récit où il est souvent question du souvenir vivace des pluies d'Afrique centrale, à l'instar d'effets plus classiques mais efficaces comme l'enregistrement du bruit des clapots du Fleuve; mais il manque complètement son but. Le public, interdit, cherche à comprendre le motif de cette brusque rupture de tonalité lorsque le rideau de fond de scène se lève, découvrant des rangées de chaises en plastique vides disposées face au public au-dessus desquelles sont suspendus de grands et bas néons, certains gisants aussi à terre. Trempé, dépenaillé et hagard, l'acteur, après avoir cajolé le cadavre d'une effigie de rapace, le dépose lentement sur son pupitre et s'assoit péniblement sur une chaise, aux pieds d'une grande peluche de léopard couchée sur le flanc. Comment ne pas voir dans ce décor de chaises vides, une métonymie du Congo (la chaise en plastique en ayant détrôné toute autre dans tous les lieux de sociabilité de RDC) et dans cette peluche, le célèbre dictateur Mobutu qui avait fait de l'animal son emblème? 
L'aigle laissé pour mort qu'étreint tendrement et gravement le Père Grégoire symbolise peut-être Saint-Jean l'Evangéliste. Comment interpréter cet étrange tableau? Le personnage hébété semble en tous cas errer dans un Congo ravagé par les guerres et les dictatures, et déserté par la foi qu'il s'efforce pourtant de ressusciter ${ }^{4} ? . .$.

7 Quelle qu'en soit l'interprétation qu'on voudra bien lui donner, cette scène apocalyptique, où la surenchère d'effets scéniques fortement sensoriels (cacophonie, explosion de lumière, trombes d'eau sur le plateau et humidité diffuse dans la salle) le dispute à un symbolisme pesant, vient singulièrement trancher avec le reste, pour ne pas dire déparer la justesse du ton de l'ensemble.

8 Cette rupture n'est pourtant pas de nature à gâcher ce spectacle dont l'on retiendra surtout la performance remarquable du comédien qui parvient non seulement à capter l'attention du public, mais plus encore à le tenir en haleine avec ce long récit d'un missionnaire, personnage qui semble désormais très éloigné des sensibilités contemporaines. Une gageure, en somme, dans une Europe occidentale qui tourne de plus en plus le dos à la religion chrétienne et a fortiori, sur un marché de l'art où prévalent d'abord et avant tout les critères de provocation et de transgression ${ }^{5}$.

9 Le sujet n'est en effet pas des plus vendeurs en ces temps où domine le paradigme de la « résistance » comme le note Pierre Halen ${ }^{6}$. Pourtant l'auteur, qui ambitionne par cette pièce de renouveler notre regard sur les missionnaires ${ }^{7}$, fait du Père Grégoire un hérault (et non un "héros" comme il s'en défend ${ }^{8}$ ) de la tradition d'un certain humanisme chrétien qui, sans se réclamer d'une quelconque pensée militante, sait faire fi des conventions et autres préceptes de l'Église qu'il juge rétrogrades. C'est un prêtre résolument moderne qui nous est peint, ce qui, forcément, nous le rend plus sympathique qu'un missionnaire père blanc pourrait a priori l'être : il distribue des préservatifs, reconnait et condamne l'existence de la pédophilie et des abus sexuels en tous genres chez ses confrères, ce qui le conduit à une vive et pragmatique critique du célibat des ecclésiastiques... Eu égard à son intention initiale, faut-il y voir une certaine complaisance de l'auteur qui, dans l'optique de favoriser l'empathie du public pour le personnage du missionnaire, le présenterait sous un jour faste, s'efforçant de raboter tout aspect dérangeant?

Il me semble que non car David Van Reybrouck n'épargne pas au public le paternalisme où son engagement prend naturellement racine : "Je l'ai fait pour les Africains ${ }^{9}$ ». De même, il ose proférer un propos qui résonne aujourd'hui comme blasphématoire "Lumumba était un salaud $»^{10}$ - mais qui s'explique de la part d'un missionnaire blanc installé au Congo à la veille de l'indépendance. La mention de ces travers idéologiques qui mettent mal à l'aise le spectateur contemporain anti-colonial, sont importants précisément parce qu'ils nous permettent de mieux saisir les enjeux politiques de l'époque : ce qui se jouait entre Africains et Européens à la fin des années 1950 et le racisme qu'impliquait l'ordre colonial auquel participait pleinement l'entreprise missionnaire.

11 Néanmoins, le plus troublant n'est point d'entendre le personnage claironner des stéréotypes racialisants (qui, du reste, sont en proportion raisonnable dans le texte), mais de se surprendre à éprouver une certaine empathie pour le personnage du missionnaire que d'aucuns se figurent n'être qu'un infâme prosélyte, abusant de son pouvoir et méprisant les cultures locales.

12 Un des moyens les plus efficaces déployés par l'auteur pour parvenir à ses fins, c'est la confrontation de deux personnages d'agents européens au service de la lutte contre la 
pauvreté dans l'outre-mer, figures majeures et historiques de l'engagement humanitaire au Tiers-monde: le missionnaire et le «travailleur humanitaire». Ce dernier - c'est-à-dire celui qui, engagé par telle $O N G$, s'en va en Afrique ou dans toute autre région "sous-développée ", aider les populations locales -, correspond à une pratique beaucoup plus socialement acceptable, surtout en Europe où l'expérience humanitaire est devenue presque un passage obligé sur le chemin de l'initiation du jeune adulte. Le Père Grégoire relate sa rencontre avec l'un d'eux venu au Kivu en "mission humanitaire» ainsi que le veut l'usage en vigueur. Il commence par rapporter les reproches formulés par son interlocuteur qui considère qu'il ne fait « pas vraiment de l'humanitaire $»^{11}$, puis il le raille en comparant la teneur de l'expérience du travailleur humanitaire à la profondeur de son engagement de missionnaire. Le temps long passé sur place (qui se compte non en semaines ou en mois mais en dizaines d'années successives) lui a non seulement permis de réaliser des projets de grande envergure - apprendre le braille pour l'enseigner à l'un de ses paroissiens ou mettre sur pied une fabrique locale de fauteuils roulants pour les estropiés de guerre ${ }^{12}-$, mais également d'apprendre à connaître les Congolais, à maîtriser leurs langues et, ainsi, à vivre et travailler avec eux.

13 Ce qui par-dessus tout, nous le rend sympathique, c'est le dévoilement progressif de sa propre humanité, c'est l'aveu de ses faiblesses qu'il nous livre tantôt avec gravité (la spirale de l'alcoolisme les soirs de solitude), tantôt avec légèreté et humour (les hilarantes séances d'absolution collective ou encore ses dysfonctionnements intestinaux), ce sont les confessions intimes, les déclarations d'amour à ses proches disparus ou éloignés par les distances et les circonstances. Ce partage d'une intimité à la fois sincère et pudique participe grandement à l'empathie du public. Les jeux d'interaction avec les spectateurs y contribuent aussi, d'autant que ce procédé est très habilement dosé. Si Vanden Broecke habite pleinement son personnage, il n'en est pas pour autant hermétique aux réactions de la salle et, à plusieurs reprises, il improvise très justement à partir de ce qu'il perçoit du public ${ }^{13}$.

Les hésitations et silences dont il ponctue son discours traduisent merveilleusement le " flux de pensée » nécessairement non linéaire du personnage. Ces interruptions qui confèrent au récit toute sa tessiture et sa chaleur, Bruno Vanden Broecke les donne à entendre dans le souffle, l'inflexion de la voix, ses légers tics de langage et quelques particularités de prononciation dues au fait que le français n'est pas la langue première du personnage ni du comédien, autant de marques de subjectivité qui nous donnent l'impression d'entendre un témoignage autobiographique plutôt qu'un monologue de fiction théâtrale.

Le personnage du missionnaire apparaît donc ici comme une figure intermédiaire ou liminale, celle d'un autre semblable (« autrement même » pour paraphraser le titre de la collection de littérature coloniale de L'Harmattan ${ }^{14}$ ) qui, depuis cette marge de l'énonciation - entre ici et là-bas - nous parle de cet ailleurs lointain, de l'Afrique, du Congo et des Congolais. En dépit de l'usage d'expressions essentialisantes appartenant à son univers référentiel daté - «Le Noir ", «L'Africain est comme ci ou comme ça » et du récit des catastrophes qui affligent le pays (guerres, massacres et autres violations) et qui peuvent à première vue paraître nous en éloigner, l'auteur parvient à nous en rapprocher. C'est en effet bien de vies humaines dont il nous parle avant tout, de vies vécues dans les joies et les peines les plus ordinaires avec leur cortège d'amours, d'amitiés, de jalousies, d'humour, de complicité etc. 
Mais c'est aussi et surtout de nous qu'il nous entretient, nous qui vivons ici dans cette Europe prospère, et à qui il est venu parler. Y sont justement dépeints les travers de nos contemporains, à commencer par l'impatience et la vitesse, l'avidité obsessionnelle et la compétitivité : " Ils veulent tout, tout de suite et provisoirement! Alors, c'est sûr, c'est pas facile, hein... $»^{15}$. Du refus de faire des choix décisifs aujourd'hui, de l'incapacité à s'engager, il en est question à plusieurs reprises dans le monologue de Père Grégoire ; le fait est que c'est une des préoccupations de David Van Reybrouck qui déclare : «J'ai 36 ans, j'appartiens à une génération qui change de métier tous les trois ans et de femme tous les quatre ans. Une génération de l'abondance qui ne fait plus de choix fondamental. C'est fascinant de voir la liberté qui naît d'un choix et de renoncements radicaux $»^{16}$. Mais si le personnage répète, par contraste avec cette tendance, "nous on a choisi, hein ", ce n'est pas pour s'ériger en modèle et faire du choix un acte définitif et imprescriptible ; bien au contraire, il conçoit le choix comme une direction que l'on ne doit pas se lasser de reprendre : « un choix, c'est un écho qui s'intensifie. À chaque fois un peu plus fort. On ne choisit pas une fois, on choisit tant de fois. Et à la fin, on appelle ça une vie $»^{17}$.

17 Au cœur du propos du Père Grégoire, c'est finalement la grande question du sens de la vie qui est posée, celle qui, selon David Van Reybrouck, est aussi celle de l'écrivain ${ }^{18}$. D'après le Père, c'est l'angoisse de la mort et du vide, l'absence de sens qu'elle entraîne pour l'athée qui le conduit à une telle frénésie : « On ne peut aimer la vie que si l'on ne trouve pas grave de mourir. On ne peut donner que quand on n'a pas trop à perdre soimême. Sinon, on s'accroche comme une moule à un bateau qui s'en ira quand-même ${ }^{19}$.

\section{NOTES}

1. David Van Reybrouck, Mission suivi de L'âme des termites, Arles, Actes Sud Papiers, 2011. Pièces traduites par Monique Nagielkop, d'après les textes originaux en néerlandais, Missie (écrit et mis en scène en 2007 au KVS de Bruxelles par Raven Rüell avec Bruno Vanden Broecke) et Die Siel van die Mier (écrit en 2004).

2. Ainsi que l'attestent les critiques belges, le spectacle qui a récolté un succès certain lors de la tournée nationale, a aussi été l'occasion de débats dans le milieu intellectuel belgo-congolais ainsi que l'illustre le montage vidéo datant de 2009 qui accompagne cette critique de Colette Braeckmann dans Le Soir en ligne : http://mad.lesoir.be/scenes/24307-missie/

3. Sur ce phénomène éditorial de grande ampleur, voir la contribution collective de la rédaction de la revue Études Littéraires Africaines qui consacre sa rubrique «à propos » à ce livre et à son auteur: Delas D., Martin-Granel N., Le Lay M., Halen P., Jewsiewicki B., Mbwiti P. M., Manga L., Chanda T. et Van Reybrouck D., "À propos de Congo. Une histoire de David Van Reybrouck », Études Littéraires Africaines, $\mathrm{n}^{\circ}$ 35, 2013, p. 119-146.

4. « Je voulais aller là où Jésus était le moins connu »: David Van Reybrouck, Mission, op.cit., p. 17.

5. Olivier Neveux, Politiques du spectateur. Les enjeux du théâtre politique aujourd'hui, Paris, La Découverte, coll. Cahiers libres, 2013. Voir notamment le chapitre 3, «Politiques de la transgression », p. 51-83. 
6. Pierre Halen et François Guiyoba, Introduction, Études Littéraires Africaines, $n^{\circ} 35$, éds. Pierre Halen et François Guiyoba, «L'impact des missions chrétiennes sur la constitution des champs littéraires nationaux en Afrique », p. 7-88.

7. "J'avais envie, raconte David Van Reybrouck, de redresser l'image de ces missionnaires ridicules, ringards, incarnant le passé colonial. Cette vision caricaturale nie l'engagement de ces hommes et femmes » : David Van Reybrouck, propos recueillis par Guy Duplat, «La position du missionnaire ", La Libre Belgique, Lalibre.be, 14/12/2007 (Repris dans la brochure de Mission au TNBA).

8. Mission, p.

9. Id., p. 17 .

10. Id., p. 20.

11. David Van Reybrouck, Mission, op.cit., p. 33.

12. "Avant les missionnaires prenaient trois ans, juste pour apprendre la langue. Aujourd'hui les gens des ONG comme MSF ou Action Contre la Faim, ils viennent travailler trois mois! Ou six mois, s'ils ont de la chance. Mais ça, c'est... c'est du tourisme, ça ! Et ils osent nous dire (...) 'ce que vous faites, ce n'est quand même pas vraiment de l'humanitaire » : ibid.

13. Par exemple, lorsqu'il rebondit sur le rire sonore et impromptu d'une jeune fille pour aborder le sujet de ses retrouvailles avec son ex-petite amie.

14. Ainsi que le soulignent Pierre Halen et François Guiyoba, art.cit., p. 8.

15. Adapté du texte original en traduction française : «Mais non, ils veulent tout, tout de suite et encore bien. Et de préférence, pas pour toujours » : David Van Reybrouck, Mission, op.cit., p. 45-46. 16. David Van Reybrouck, propos recueillis par Guy Duplat, «La position du missionnaire », art.cit.

17. David Van Reybrouck, Mission, op.cit., p. 43, repris sur la $1^{\text {ère }}$ de couverture de la brochure de Mission au TNBA.

18. «L'engagement des missionnaires me rappelle, souligne David Van Reybrouck, celui de l'écrivain dans l'écriture. Finalement le sens du spectacle, c'est aussi ça : la recherche du sens » : David Van Reybrouck, propos recueillis par Guy Duplat, art. cit.

19. David Van Reybrouck, Mission, op.cit., p. 46.

\section{AUTEUR}

\section{MAËLINE LE LAY}

Chargée de recherche, laboratoire LAM (Les Afriques dans le monde, CNRS), Bordeaux 